A

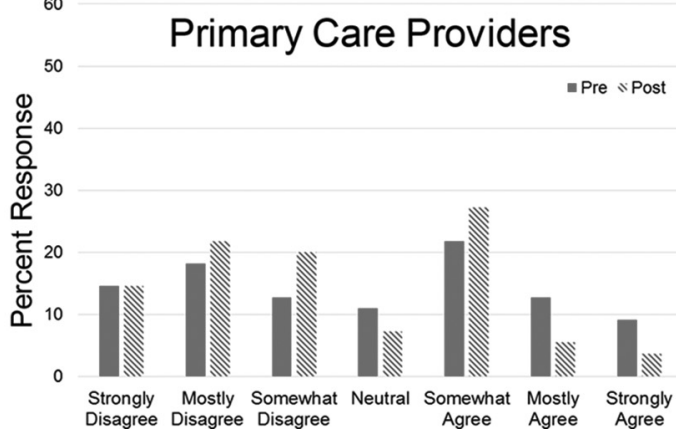

B

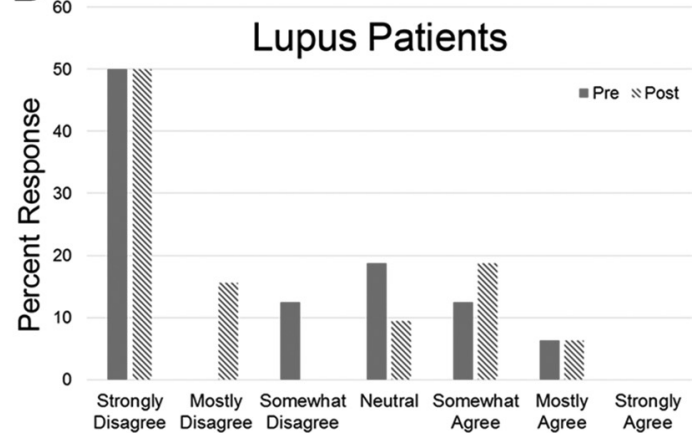

Abstract 100 Figure 1 Perceived Impact of Poverty and Study Protocol Adherence. A) Primary care providers were asked to respond to the following statement Indigent patients are less likely to comply with a study protocol before and after the educational program and had a shift towards increased disagreement (median [interquartile range], Pre $4[2,5]$ and Post $3[2,5], p=0.024)$ ). B) Similarly lupus patients were asked to respond to I think people who are poor or have less education are less likely to correctly follow the instructions they are given during a research study with a majority of responses disagreeing which remained stable after the program (Pre 2 [1, 4] and Post 1.5 [1, 4.75], $p=0.72$ ). Significant differences in responses between providers and patients were noted both before the program $(p=0.0037)$ and after the program $(p=0.0088)$. The shift in providers response towards disagreement after the program was not large enough to result in post-test responses similar to the patients.

Results 55 providers and 32 lupus patients completed the questionnaires and educational program. Knowledge topics included 1) triggers of SLE, 2) lupus racial differences, and 3) informed consent. There was a statistically significant difference between PCPs and patients on the pre-test informed consent topic (PCP 46\% correct and patient 15\%, p=0.0005). Post-test results were similar between groups. The education program resulted in improvement in knowledge scores for PCPs (Q1 $42 \%$ to $85 \%, \quad \mathrm{p}<0.001$; Q2 $56 \%$ to $91 \%$, $\mathrm{p}<0.0001 ; \mathrm{Q} 384 \%$ to $87 \%, \mathrm{p}=0.5$ ) and patients (Q1 $38 \%$ to $84 \%, \mathrm{p}=0.0003$; Q2 $56 \%$ to $75 \%, \mathrm{p}=0.1$; Q3 $47 \%$ to $72 \% \mathrm{p}=0.01)$. Topics of clinical trial belief questions included perceived risks of trials, differing racial background between provider and patient, education level needed for trial participation, and perceived effect of poverty on protocol compliance. The final topic demonstrated the greatest incongruence between PCPs and patients (figure 1).

Conclusions Beliefs about race, education, and poverty may impact referral to clinical trial centers and clinical trial participation. More than a third of PCPs believe that indigent people are poor candidates for clinical trials. Despite a shift away from this attitude following an educational program, providers remained much more likely than lupus patients to hold this opinion.

Funding Source(s): HHS-OMH-CPI-MP-17-002-1

\section{D-MANNOSE AMELIORATES SYSTEMIC LUPUS ERYTHEMATOSUS-LIKE DISEASE IN B6.MRL/LPR MOUSE MODEL}

${ }^{1}$ Haiting Wang*, ${ }^{2}$ Xiangyu Teng, ${ }^{2}$ Georges Abboud, ${ }^{2}$ Wei Li, ${ }^{2}$ Laurence Morel. ${ }^{1}$ Renji Hospital South Campus, Shanghai JiaoTong University School of Medicine, Shanghai, China; ${ }^{2}$ Department of Pathology, Immunology, and Laboratory Medicine, University of Florida, Gainesville, FL, USA

\subsection{6/lupus-2019-Ism.101}

Background Systemic lupus erythematosus (SLE) is an autoimmune disease that is characterized by high morbidity and mortality and its treatment remains challenging. Inflammatory dendritic cells (DCs) have been shown to participate in the initiation and perpetuation of lupus pathogenesis, and tolerogenic DCs have a potential for cell-based therapy in this condition. The mannose receptor (MR, CD206) is a C-type lectin expressed by DCs and its cross-linking induces anti-inflammatory immunosuppressive effects. D-Mannose is a C-2 epimer of glucose that exhibit immunoregulatory effect in models of autoimmune diseases, such as type 1 diabetes and lung airway inflammation. However, the function of D-Mannose treatment in lupus remains unknown.

Methods B6.MRL-Fas(lpr) (B6.lpr) mice at 4 months of age were treated with D-Mannose in drinking-water for 2 months. Autoantibody production and immune cell activation were compared between the two groups. In vitro, GMCSF bone marrow-derived dendritic cells (BMDCs) from non-autoimmune B6 mice were cultured for 5 days to generate mature dendritic cells. On day 5, BMDCs were treated with $10 \mathrm{mM}$ glucose (G10) or $10 \mathrm{mM}$ Mannose (M10) for 24 hour and pulsed with LPS for an additional 4 hour. Surface markers and cytokines secretion of BMDCs were analyzed.

Results The D-Mannose treatment significantly decreased serum anti-dsDNA antibody at week 4. It also increased the percentage of naïve $\mathrm{T}(\mathrm{Tn})$ cells and decreased CD4 + T cell activation measured as CD44 +expression. Follicular helper T (TFH) cells/follicular regulatory $\mathrm{T}$ (TFR) cells ratio was reduced after D-Mannose treatment. The low frequency of regulatory $\mathrm{T}$ (Treg) cells in $\mathrm{B} 6 / \mathrm{lpr}$ mice was also expanded after treatment. Besides, D-Mannose treatment increased CD206 expression on spleenic DCs. In vitro experiments showed that D-Mannose promoted a tolerogenic phenotype in BMDCs by decreasing the expression of activation markers (CD40, CD80, CD86) and promoting that of inhibitory markers (CD206 and CD64) expression in B6 mice. Additionally, D-Mannose reduced inflammatory cytokine secretion in BMDCs.

Conclusions D-Mannose ameliorates the development of lupuslike disease in the B6/lpr mouse model, which may be due to the induction of tolerogenic DCs.

Funding Source(s): National Key R and D Program of China (2017YFC0909000) 
A

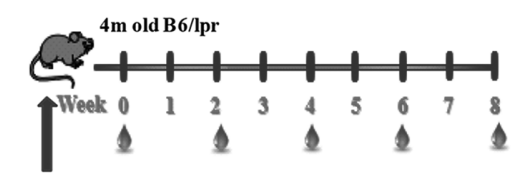

$\mathrm{D}-\mathrm{M}$ annose $: 1.1 \mathrm{M}$ d isso lved in drinking w ater Con tro l: sterile drinking w ater

D

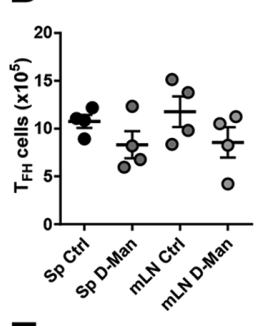

$E \quad F$
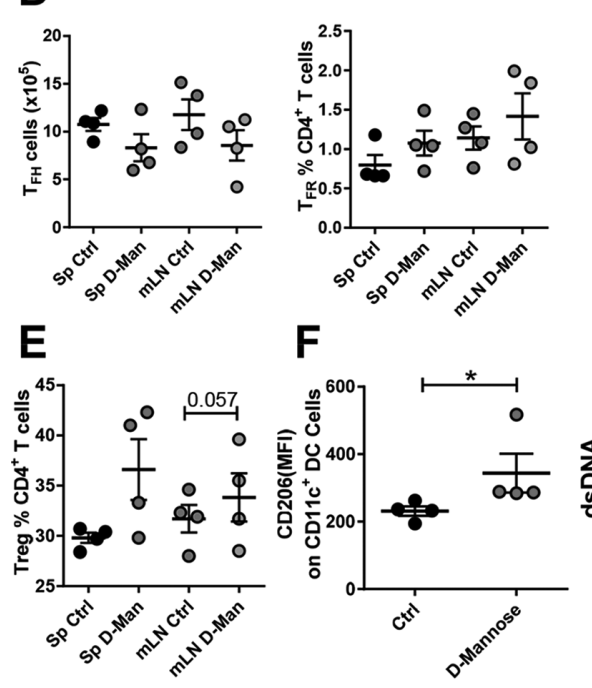

$F$
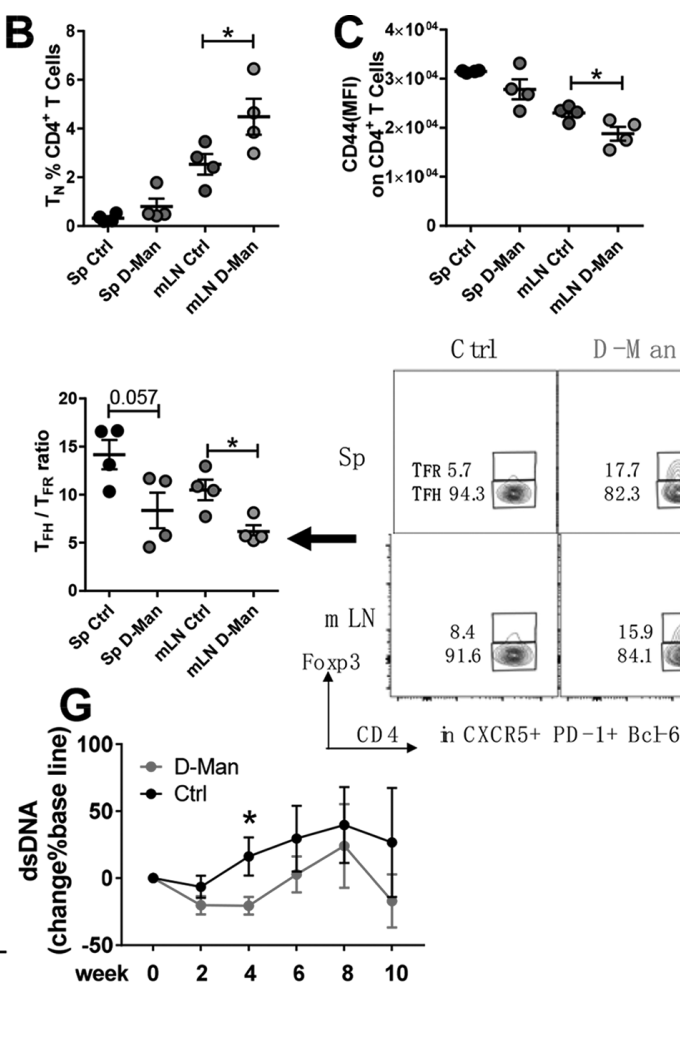

CD80
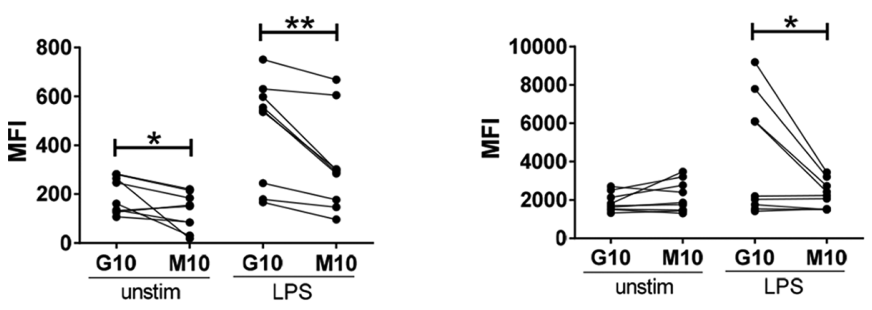

CD32b
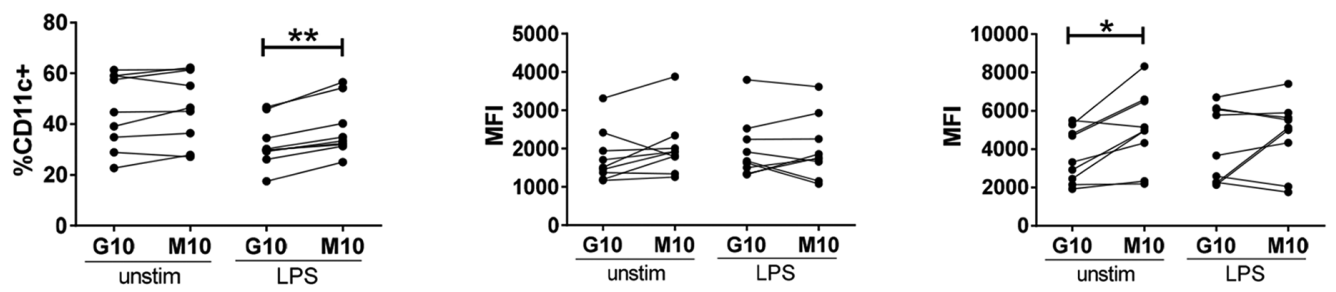

J TNF- $\alpha$

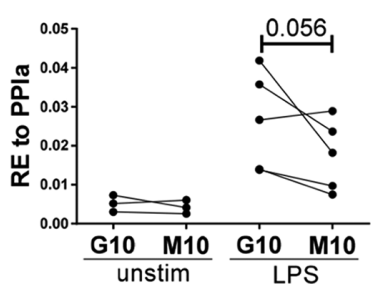

BAFF

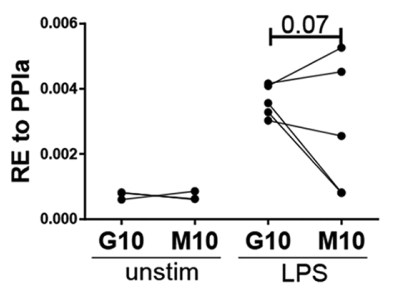

CD86

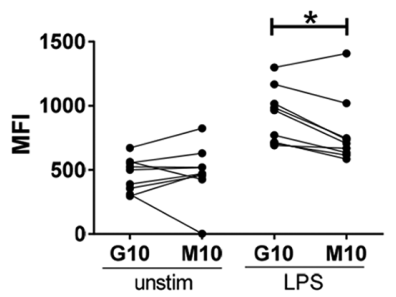

CD64

Mx-1

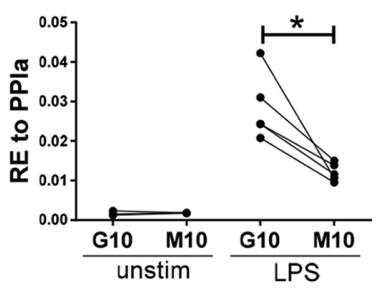

Abstract 101 Figure 1 D-Mannose ameliorates systemic lupus erythematosus-like disease in B6.MRLIpr mouse model. (A) In vivo study design (B) Frequency of CD4+ CD44- CD62L+ Tn cells (C) Expression of CD44 on CD4+ T cells (D) Frequency of PD-1hi CXCR5hi BCL6+ Foxp3Tfh and PD-1hi CXCR5hi BCL6+ Foxp3+ Tfr subsets (E) Frequency of CD4+ Foxp3+ Treg subsets (F) Expression of CD206 on CD11c+ spleen DCs (G) Serum anti-dsDNA IgG in B6.Ipr mice (H) Expression of activation markers (CD40, CD80, CD86) on CD11C+ BMDCs (I) Expression of inhibitory markers (CD206, CD32b, CD64) on CD11C+ BMDCs (J) Inflammatory cytokine levels of BMDCs 